\section{ces}

We found that

protocells

made from

these more

complex mix-

tures actually

require condi-

tions akin to

alkaline hydro-

thermal vents

to form at all

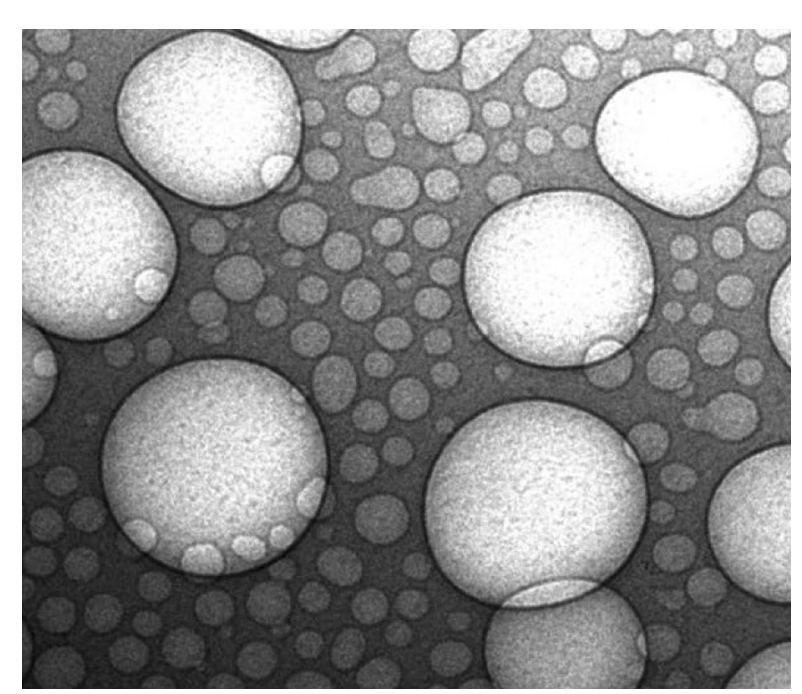

Image reprinted from Jordan et al. (2019), Springer Nature Limited.

Membranes were probably involved in the origin of life. But the most common models of primordial membranes are unstable towards the high temperatures and salinities assumed by many to be involved. Now, writing in Nature Ecology \& Evolution, researchers from University College London led by Nick Lane have shown that model membranes with more different types of component not only tolerate alkaline hydrothermal conditions but in fact benefit from them.

Modern cell membranes are formed from lipids whose structures have been optimized by eons of evolution. Conversely, prebiotically relevant synthetic processes, such as hydrothermal Fischer-Tropsch chemistry, yield much simpler singlechain amphiphiles (SCAs) bearing hydroxyl or carboxyl head groups. While SCA vesicles offer invaluable insight into the possible behaviour and composition of 'protocells' simple systems thought to precede the first living cells - they are sensitive to environmental conditions. They are

\title{
Mixed up membranes protect protocells
}

widely held to be incompatible with popular abiogenesis models involving submarine hydrothermal vents. These are warm, alkaline environments, containing millimolar concentrations of salts including divalent cations - all of which are unfavourable for the formation of SCA membranes.

"This has even led some researchers to call for an abandonment of research into any possible origin of life scenario based in the ocean," says Sean Jordan, first author of the study.

Confronted with this problem, the team - leading advocates of hydrothermal vent scenarios asked whether more heterogeneous membrane compositions would prove more stable to extremes of heat, salinity and $\mathrm{pH}$. Typical model membranes consist of one to three SCAs. Previous work by Szostak and colleagues showed that mixtures of long- and short-chain lengths can promote vesicle formation even under dilute conditions. It seems clear that while simple membrane compositions offer many advantages, more complex mixtures can challenge conventional wisdom.

To this end, the group first increased the number of different species so that the membranes were formed from mixtures of up to 12 different SCAs. Increasing the diversity of the membrane composition imparted stability across a much wider $\mathrm{pH}$ range. Furthermore, at high $\mathrm{pH}$, these mixtures tolerated concentrations of sodium chloride, or of divalent cations, comparable to those found in modern oceans. This is presumably because at high $\mathrm{pH}$ such cations form hydroxides rather than chelating to carboxylate moieties in the SCAs and thereby promoting precipitation. However, the combination of high $\mathrm{pH}$, high salinity and divalent cations still led to aggregation and precipitation of the vesicles.

Biology provided an inspired solution: isoprenoids, which are found in archaeal phospholipids. "Isoprenoids have been studied very little," says Lane, "but the fact that they are the basis of cell membranes in archaea would imply they arose early on, and there are good reasons to attempt prebiotic studies of them." Adding two isoprenoids, geranic acid and geraniol, yielded vesicles that were stable under even the harshest of conditions.

"We found that protocells made from these more complex mixtures actually require conditions akin to alkaline hydrothermal vents to form at all," says Lane. The high temperatures were required to melt long-chain SCAs. Meanwhile, the high $\mathrm{pH}$ aided dissolution of fatty acids by deprotonation and reduced the detrimental effect of divalent cations by hydroxide formation. Although oceans on the early Earth are not widely thought to be strongly alkaline, $\mathrm{pH}$ can reach as high as 9-11 within hydrothermal vents. This is sufficiently alkaline to promote fatty acid dissolution and vesicle formation from these SCA mixtures.

The prebiotic plausibility of isoprenoids remains an open question. "I'm not aware of any successful prebiotic syntheses, but at the same time I'm not aware of anyone who has tried in a serious way," says Lane. But it may be that the benefits of isoprenoids originate in their branched, unsaturated structures - which can also be found in simpler and arguably more relevant fatty acids. If so, this stabilizing mechanism may prove to be quite general.

Andrew Bissette, Editor, Communications Chemistry

ORIGINAL ARTICLE Jordan, S. F. et al. Promotion of protocell self-assembly from mixed amphiphiles at the origin of life. Nat. Ecol. Evol. 3, 1705-1714 (2019) RELATED ARTICLE Butin, I. et al. Chain-length heterogeneity allows for the assembly of fatty acid vesicles in dilute solutions. Biophys. J. 107, 1582-1590 (2014) 